\title{
Revista Española de
} Nutrición Humana y Dietética

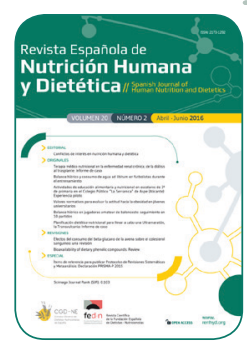

\section{REVISIÓN}

\section{Efectos del consumo del beta-glucano de la avena sobre el colesterol sanguíneo: una revisión}

\author{
Aránzazu Aparicio Vizuete ${ }^{a, *}$, Rosa María Ortega Anta $^{a}$ \\ a Departamento de Nutrición y Bromatología I (Nutrición), Facultad de Farmacia, Universidad Complutense de Madrid, Grupo de \\ Investigación VALORNUT-UCM (92030), Madrid, España. \\ *araparic@ucm.es
}

Recibido el 16 de septiembre de 2015; aceptado el 17 de noviembre de 2015.

Efectos del consumo del beta-glucano de la avena sobre el colesterol sanguíneo: una revisión

\section{PALABRAS CLAVE}

Beta-Glucanos;

Glucanos;

Avena;

Avena Sativa;

Fibra Dietética;

Fibra soluble;

Colesterol;

Colesterol LDL;

Revisión.

\section{RESUMEN}

Las cifras sanguíneas de colesterol total y de lipoproteínas de baja densidad (LDL) elevadas se consideran como uno de los más importantes factores de riesgo de enfermedad coronaria. La avena es un cereal rico en proteínas, lípidos, vitaminas, minerales y fibra soluble, entre las que se incluye el beta-glucano. Debido a las características físico-químicas del beta-glucano, se ha propuesto que la avena pudiera contribuir a disminuir las cifras de colesterol sanguíneo, así como a controlar la glucosa plasmática postprandial y la respuesta insulínica. Numerosos estudios señalan que el consumo de un mínimo de $3 \mathrm{~g} /$ día de beta-glucano de forma regular, como parte de una dieta con un bajo contenido en grasa saturada y colesterol, puede contribuir a disminuir el riesgo de enfermedad coronaria, tal y como han aprobado diversas agencias reguladoras, como la Food and Drug Administration de Estados Unidos o la Agencia Europea de Seguridad Alimentaria. 
Effects of oat beta-glucan intake on blood cholesterol: a review

\section{KEYWORDS}

Beta-Glucans;

Glucans;

Avena;

Oat;

Avena Sativa;

Dietary Fiber;

Soluble fiber:

Cholesterol;

LDL Cholesterol;

Review

\section{ABSTRACT}

Elevated total and low density lipoprotein ( $L D L)$ cholesterol levels are considered major risk factors for coronary heart disease. Oat is a cereal that is a good source of proteins, fat, minerals and vitamins, as well as soluble fiber, including beta-glucans. Due to the physicochemical characteristics of the beta-glucan, it has been proposed that oats may help reduce blood cholesterol levels and help control postprandial plasma glucose and insulin response. A large body of clinical studies suggests that the consumption of at least $3 \mathrm{~g}$ per day of oat betaglucan, as part of a diet low in saturated fat and cholesterol, may reduce the risk of coronary heart disease, which has been approved by several regulatory agencies, as the Food and Drug Administration in the USA and the European Food Safety Authority in Europe.

\section{CITA}

Aparicio Vizuete A, Ortega Anta RM. Efectos del consumo del beta-glucano de la avena sobre el colesterol sanguíneo: una revisión. Rev Esp Nutr Hum Diet. 2016; 20(2): 127 - 139. doi: 10.14306/renhyd.20.2.183

\section{INTRODUCCIÓN}

La avena (Avena sativa L.) es un cereal cuyo grano completo tiene un elevado contenido en fibra dietética soluble, en la que se incluye el beta-glucano, aportando también proteínas, lípidos, vitaminas, minerales y polifenoles, como las avenantramidas $^{1,2}$. Además, la avena no contiene gluten, de ahí que sea un cereal bien tolerado por la mayoría de las personas con celiaquía ${ }^{3,4}$.

Por su contenido en fibra y fitoquímicos, principalmente, diversos estudios clínicos han evaluado la eficacia del consumo de los cereales de grano completo, en la prevención y control de la enfermedad cardiovascular, diabetes, regulación de la presión arterial, control de peso, salud gastrointestinal, e incluso el cáncer ${ }^{1,4,5}$. En concreto, en el caso de la avena numerosas investigaciones han mostrado un efecto positivo en la reducción del colesterol en sangre y, por tanto, en la reducción del riesgo de enfermedad coronaria6,7, habiéndose atribuido este efecto al beta-glucano, más que a la fibra soluble en general ${ }^{8}$. Por ello, se han realizado diversas declaraciones de propiedades saludables aprobadas por la legislación actual en relación al consumo de productos/ alimentos elaborados con este cereal.

Se considera que la avena y otros cereales de grano completo (trigo, maíz, arroz, cebada, centeno, quinoa, mijo) deben formar parte de una dieta equilibrada, habiéndose incluido en las guías alimentarias de numerosos países europeos, asiáticos y americanos. En el año 2010 las Guías Alimentarias Americanas (Dietary Guidelines for Americans) establecieron como conveniente que al menos 3 raciones/día de los cereales consumidos fueran cereales de grano completo ${ }^{9}$ (16 g/ración), pauta que fue incorporada a las guías españolas en el año $2011^{10}$. 


\section{CARACTERÍSTICAS FÍSICO-QUÍMICAS DEL BETA-GLUCANO DE AVENA Y SU RELACIÓN CON LA REDUCCIÓN DEL COLESTEROL SANGUÍNEO}

El beta-glucano es un tipo de fibra soluble que se encuentra de forma natural en la avena y cebada, aunque también se pueden encontrar en algas y setas ${ }^{11}$.

Es un polímero lineal de unidades de glucosa unidas mediante enlaces glucosídicos $\beta$ - $(1 \rightarrow 3)$ y $\beta$ - $(1 \rightarrow 4)^{12}$, que se localiza principalmente en las paredes celulares del endospermo del grano de avena ${ }^{13}$.

El contenido en beta-glucano de la avena depende de las condiciones de cultivo y de crecimiento ${ }^{14}$. Diferentes estudios han observado una variación de 2 a 3 veces en el contenido de beta-glucano en diversos cultivos de avena ${ }^{15-17}$. Además, éste también está regulado por la enzima beta-glucano endohidrolasa $(1 \rightarrow 3,1 \rightarrow 4)$, que degrada la pared celular del endospermo durante la germinación ${ }^{18}$, o la preparación de alimentos ${ }^{19}$, y disminuye la cantidad de beta-glucano en los alimentos. Por ello, el contenido en beta-glucano en el grano de avena puede variar de un $1,8 \%$ a un $5,5 \%$, y se ha encontrado en algunas variedades de este cereal hasta un $7 \%$ del peso seco total ${ }^{20}$, aunque lo más habitual es hallar un contenido del $4,0-5,5 \%{ }^{21-23}$.

Como otros tipos de fibra soluble, en el organismo, el betaglucano forma geles viscosos en el tracto gastrointestinal, retrasando el vaciamiento gástrico e interfiriendo con la actividad de diferentes enzimas pancreáticas, lo que ralentiza los procesos de digestión y absorción de nutrientes $s^{7,23,24}$ e incrementa la excreción de ácidos biliares ${ }^{25,26}$.

Esta viscosidad se relaciona con algunos de los efectos sanitarios señalados anteriormente, como la reducción de la glucosa plasmática postprandial y la mejora de la respuesta insulínica, así como la disminución del colesterol sanguíneo $7,25,27,28$, entre otros efectos.

Tanto el peso molecular, como la conformación del betaglucano de avena, son dos factores importantes a tener en cuenta en el alimento, ya que influyen sobre las propiedades físicas del mismo, incluyendo la solubilidad y la viscosidad ${ }^{14}$ $y$, por ende, en sus efectos sobre la salud. Wolever y Cols. ${ }^{8}$, tras analizar el efecto hipocolesterolemiante de unos cereales de desayuno con beta-glucano de avena con diferentes pesos moleculares en un grupo de individuos sanos, observaron que al consumir los cereales con elevado (2.210kDa) y medio $(530 \mathrm{kDa})$ peso molecular, la concentración sérica de las lipoproteínas de baja densidad (LDL) disminuyó de forma similar en ambos casos (5\%), y fue un $50 \%$ más baja cuando el peso molecular del beta-glucano era bajo (210kDa). Diversos estudios han señalado que para que el beta-glucano de la avena produzca un impacto en la reducción del colesterol sanguíneo es necesario que éste tenga un peso molecular de al menos $1.200 \mathrm{kDa}^{29-31}$ y que este factor debe tenerse en cuenta, junto con la cantidad de beta-glucano a ingerir, a la hora de realizar estudios dirigidos a evaluar la eficacia del mismo sobre las cifras de colesterol sanguíneo $0^{6,14,23}$.

Además, el tipo de procesado y las condiciones de almacenamiento también pueden afectar a la cantidad, solubilidad y peso molecular del beta-glucano en los productos elaborados con avena ${ }^{4,14,23,32}$. La avena es un cereal que, como tal, no puede ser ingerido en crudo sino que tiene que ser procesado para hacerlo comestible ${ }^{33}$, siendo habitualmente consumido en forma de copos, gachas, cereales de desayuno, muesli, barritas, galletas y tortitas ${ }^{20}$.

Algunas investigaciones han encontrado que uno de los procesados que más mejoran la solubilidad del beta-glucano de la avena es el extrusionado. Aunque existen diferentes datos en relación con la solubilidad del beta-glucano de la avena, Tosh y Cols. ${ }^{34}$ encontraron que cuando el salvado de avena comercial era incorporado a un cereal extrusionado la solubilidad del mismo aumentaba, pasando de un 39 a un $67 \%$, lo que también coincide con lo observado por Zhang y Cols. $^{35}$. Otros procesados en los que la avena se calienta en presencia de agua, como el horneado o la cocción ${ }^{36}$, también incrementan la solubilidad del beta-glucano y su viscosidad, lo que se asocia a un aumento de su bioactividad ${ }^{8}$.

Por otra parte, diversos estudios indican que el peso molecular del beta-glucano no suele variar en los copos de avena, las gachas, el salvado de avena o las tortitas ${ }^{19}$, aunque sí parece modificarse en la elaboración del pan, pasta o galletas a base de avena ${ }^{37}$. Sin embargo, a pesar de esta reducción, estos alimentos parecen mantener los efectos positivos sobre la disminución del colesterol sanguíneo ${ }^{38}$.

Charlton y Cols. ${ }^{39}$ analizaron el efecto reductor del colesterol en sangre del beta-glucano de avena a distintas cantidades e incorporado a distintas matrices alimentarias: gachas de avena y barritas de cereales de avena con 3,2g de beta-glucano de alto peso molecular; cereales de desayuno con base de avena y barritas de cereales de arroz inflado y trigo con $1,5 \mathrm{~g}$ de beta-glucano de peso molecular medio y de alta solubilidad y cereales de desayuno de maíz y barritas de cereales de arroz inflado y trigo sin beta-glucano (grupo control). Al evaluar los resultados, encontraron una mayor reducción del colesterol total (CT) y de las LDL en los individuos que consumieron los alimentos con beta-glucano de avena, respecto a los que tomaron el alimento control. Al comparar 
el efecto hipocolesterolemiante entre los sujetos que tomaron avena se observó que éste fue similar. Los autores concluyeron que la matriz alimentaria en la que se incluye el beta-glucano, así como el peso molecular y la solubilidad del mismo, son factores importantes a tener en cuenta a la hora de observar efectos positivos de este componente de la avena sobre la salud cardiovascular en individuos con hipercolesterolemia moderada.

Además, el beta-glucano de avena aislado se emplea en la industria alimentaria como espesante y para mejorar la calidad sensorial de bebidas ${ }^{4}$. En relación a sus efectos sobre el colesterol sanguíneo, se ha observado que cuando éste se consume incluido en alimentos líquidos es más efectivo que cuando se consume incorporado en alimentos sólidos $32,40-42$. Kerckhoffs y Cols. ${ }^{37}$ realizaron dos estudios en los que analizó, por un lado, el efecto sobre el colesterol sanguíneo del beta-glucano de avena incorporado a pan y galletas con respecto a un alimento control (pan y galletas ricas en fibra de trigo) (estudio 1) y, por otro, incorporado a un zumo de naranja en comparación con una bebida control rica en fibra de trigo (estudio 2). Al comparar el efecto sobre la disminución del colesterol sanguíneo del beta-glucano entre los grupos que recibieron los alimentos con beta-glucano con respecto a los que recibieron los alimentos control, se observó que, en el estudio 1, aunque se produjeron disminuciones del colesterol sanguíneo, con respecto al inicio del estudio, en los individuos que tomaron el pan y las galletas con avena éstas no fueron significativas al compararlas con las encontradas en el grupo control. Sin embargo, en el estudio 2, la bebida rica en beta-glucano disminuyó la concentración sérica de
CT en un 3,8\% y la de LDL en un 6,7\% (estadísticamente significativa) con respecto a la bebida control. Estos resultados pusieron de manifiesto que tanto la matriz alimentaria como el procesamiento de los alimentos pueden influir en las propiedades hipocolesterolemiantes del beta-glucano de avena.

Con respecto a las condiciones de almacenamiento de los productos elaborados con avena, Beer y Cols. ${ }^{43}$ observaron que el almacenamiento por congelación de unas magdalenas con salvado de avena redujo más de un $50 \%$ la solubilidad del beta-glucano, lo que puede disminuir la solubilidad del mismo en el intestino y sus efectos bioactivos. Un reciente estudio ha mostrado que someter a unas magdalenas con beta-glucano de avena a una serie de ciclos de congelación (14 horas a $-18 \stackrel{\circ}{\circ}$ ) y descongelación (10 horas a temperatura ambiente) reduce progresivamente la solubilidad del beta-glucano, de forma que tras cuatro ciclos ésta puede disminuir en un $50 \%$.

\section{MECANISMOS DE ACCIÓN PROPUESTOS PARA EL EFECTO HIPOCOLESTEROLEMIANTE DEL BETA-GLUCANO}

Se han propuesto diversos mecanismos por los que el beta-glucano podría ejercer su efecto reductor del colesterol sanguíneo, haciéndolo biológicamente plausible 32,45 (Tabla 1) 4,24,46,47.

Tabla 1. Mecanismos del beta-glucano implicados en la regulación de la homeostasis del colesterol (adaptado de Chen y Huang ${ }^{45}$ ).

\begin{tabular}{|c|c|c|}
\hline Referencia & Mecanismo & Efectos \\
\hline Erkkila y Col. ${ }^{24}$ & $\begin{array}{l}\text { Formación de geles viscosos en el tracto } \\
\text { gastrointestinal }\end{array}$ & $\begin{array}{l}\text { Disminución de la absorción de colesterol dietético y } \\
\text { aumento de la excreción de ácidos biliares }\end{array}$ \\
\hline Sadiq Butt y Cols. ${ }^{4}$ & $\begin{array}{l}\text { Disminución de la reabsorción de ácidos } \\
\text { biliares }\end{array}$ & $\begin{array}{l}\text { Aumento de la conversión de colesterol sanguíneo en } \\
\text { ácidos biliares y disminución del colesterol sanguíneo }\end{array}$ \\
\hline Ellegard y Col. ${ }^{46}$ & Aumento de la actividad de la CYP7A1 & $\begin{array}{l}\text { Incremento de la conversión del colesterol en ácidos } \\
\text { biliares }\end{array}$ \\
\hline Reihner y Cols. ${ }^{47}$ & $\begin{array}{l}\text { Aumento de los receptores de } \\
\text { LDL-colesterol }\end{array}$ & $\begin{array}{l}\text { Aumento del transporte del colesterol sanguíneo a los } \\
\text { hepatocitos }\end{array}$ \\
\hline Ellegard y Col. ${ }^{46}$ & $\begin{array}{l}\text { Incremento de la actividad de la } \\
\text { HMG-CoA reductasa }\end{array}$ & Aumento de la síntesis de colesterol \\
\hline
\end{tabular}

CYP7A1: colesterol 7- $\alpha$-hidroxilasa; HMG-CoA reductasa: 3-hidroxi-3-metilglutaril coenzima A reductasa 
Éste, como otras fibras solubles, forma geles viscosos en el tracto gastrointestinal y puede disminuir la absorción del colesterol dietético y la reabsorción de los ácidos biliares ${ }^{24}$. La inhibición de la reabsorción de los ácidos biliares aumenta la síntesis de estos ácidos biliares a partir del colesterol sanguíneo, disminuyendo así la cantidad de colesterol LDL sérico ${ }^{4}$.

Por otro lado, la disminución de los ácidos biliares a nivel hepático puede aumentar la actividad de la enzima colesterol 7-a hidroxilasa, lo que podría producir un incremento de la síntesis de ácidos biliares a partir del colesterol hepático, así como aumentar la síntesis de los receptores hepáticos para las LDL y verse disminuida la circulación sanguínea de las mismas $^{46,47}$. Además, la disminución del colesterol hepático puede estimular la actividad de la enzima 3-hidroxi-3-metilglutaril coenzima $A$ reductasa, lo que podría provocar un aumento de la síntesis de colesterol en sangre ${ }^{46}$ y contribuir a reducir las LDL circulantes.

\section{EFECTO REDUCTOR DEL COLESTEROL SANGUÍNEO ASOCIADO AL CONSUMO DE BETA-GLUCANO DE AVENA}

La enfermedad coronaria es una de las principales causas de morbilidad y mortalidad a nivel mundial ${ }^{48}$. Las actuales directrices para el tratamiento de la dislipemia señalan que existe una importante relación entre el CT y las LDL con el riesgo de enfermedad cardiovascular ${ }^{49-51}$ y, que algunas modificaciones en el estilo de vida, como la inclusión del consumo de fibra soluble en la dieta, pueden contribuir a disminuir el riesgo del padecimiento de la misma ${ }^{49,52,53}$. Uno de los medicamentos más utilizados en el tratamiento de la enfermedad cardiovascular son las estatinas, las cuales son muy eficaces disminuyendo la concentración sérica de las LDL. La modificación del estilo de vida, en la que se incluye la modificación de la dieta, debe estar siempre presente junto al tratamiento farmacológico, y algunas personas pueden necesitar algo más para conseguir resultados satisfactorios, habiéndose propuesto que el beta-glucano podría contribuir a mejorar el efecto de las estatinas ${ }^{45}$.
Actualmente, las directrices europeas señalan que el consumo de 5-15g/día de fibra soluble procedente de alimentos con avena podría ser beneficioso ${ }^{49}$, mientras que la Food and Drug Administration de Estados Unidos aconseja el consumo de $10-25 \mathrm{~g} /$ día procedente de la avena u otros alimentos ${ }^{50}$.

Hoy en día existen numerosos alimentos funcionales para reducir las cifras de colesterol sanguíneo, entre los que se incluyen los que contienen beta-glucano de avena ${ }^{32,54}$. Las agencias de seguridad alimentaria de muchos países han aprobado alegaciones de salud en relación al papel de la fibra soluble de los alimentos con avena o del beta-glucano de la misma en la reducción del colesterol en sangre, como la de Estados Unidos ${ }^{28}$, Canadá55, Europa ${ }^{27}$, Australia y Nueva Zelanda ${ }^{56}$ y Malasia ${ }^{57}$. Asimismo, el Joint Health Claims Initiative del Reino Unido reconoce la importancia de incluir el betaglucano de avena en la dieta de la población para ayudar a disminuir el colesterol en sangre y el riesgo de enfermedad coronaria $^{58}$. Excepto en el caso de Malasia, estas alegaciones están basadas en un consumo regular de $3 \mathrm{~g} /$ día de beta-glucano, aunque en el caso de Estados Unidos los alimentos con avena deben contener al menos $0,75 \mathrm{~g}$ de beta-glucano por ración y en el de Europa $1 \mathrm{~g}$ por ración ${ }^{6}$.

Numerosos estudios han investigado los efectos beneficiosos del beta-glucano de avena en la reducción del colesterol en sangre, aunque los resultados de los mismos, en ocasiones, son contradictorios. Esto podría deberse, entre otros, a los diferentes diseños de los estudios, al tamaño de las muestras empleadas, a la cantidad de beta-glucano utiliza$\mathrm{da}$, a la matriz alimentaria en la que se incorpora este ingrediente 0 al peso molecular del mismo $6,7,14$.

En la Tabla 2 se muestran los resultados de diversos estudios de intervención realizados en sujetos con/sin hipercolesterolemia, publicados en inglés en los últimos 15 años, en los que se ha analizado el efecto reductor sobre el colesterol sanguíneo tras el consumo de alimentos con avena, consultando las bases de datos de Pubmed y Cochrane Library, utilizando las palabras clave "oat", "oat $\beta$-glucan" y "blood colesterol", e incluyendo estudios con una duración mínima de 2 semanas y una cantidad de beta-glucano $\geq 3 \mathrm{~g} /$ día $8,37,38,39,41,42,59-79$.

Tabla 2. Características de los estudios.

\begin{tabular}{|c|c|c|c|c|c|c|c|}
\hline Referencia & $n$ & $\begin{array}{l}\text { Tipo de } \\
\text { estudio }\end{array}$ & $\begin{array}{l}\text { Duración } \\
\text { del estudio } \\
\text { (semanas) }\end{array}$ & $\begin{array}{c}\text { Lípidos sanguíneos } \\
\text { medios al inicio del } \\
\text { estudio (mmol/L) }\end{array}$ & $\begin{array}{l}\text { Producto de } \\
\text { intervención/ } \\
\text { control }\end{array}$ & $\begin{array}{c}\text { Cantidad de } \\
\text { beta-glucano } \\
\text { (g/día) }\end{array}$ & $\begin{array}{l}\text { Cambios en } \\
\text { los lípidos } \\
\text { sanguíneos* }\end{array}$ \\
\hline $\begin{array}{l}\text { Lovegrove y } \\
\text { Cols. }^{59}\end{array}$ & 62 & Paralelo & 12 & $\begin{array}{l}\text { CT: } 6,4 \\
\text { LDL: } 4,30 \\
\text { HDL: } 1,5 \\
\text { TG: } 1,5\end{array}$ & $\begin{array}{c}\text { Avena } \\
\text { (beta-glucano) } \\
\text { en yogur bajo en } \\
\text { grasa/salvado } \\
\text { de trigo en yogur } \\
\text { bajo en grasa }\end{array}$ & 3 & $\begin{array}{l}\leftrightarrow \mathrm{CT} \\
\leftrightarrow \mathrm{LDL} \\
\leftrightarrow \mathrm{HDL} \\
\leftrightarrow \mathrm{TG}\end{array}$ \\
\hline
\end{tabular}


Rev Esp Nutr Hum Diet. 2016; 20(2): 127 - 139

\begin{tabular}{|c|c|c|c|c|c|c|c|}
\hline Referencia & $\mathrm{n}$ & $\begin{array}{l}\text { Tipo de } \\
\text { estudio }\end{array}$ & $\begin{array}{l}\text { Duración } \\
\text { del estudio } \\
\text { (semanas) }\end{array}$ & $\begin{array}{l}\text { Lípidos sanguíneos } \\
\text { medios al inicio del } \\
\text { estudio (mmol/L) }\end{array}$ & $\begin{array}{l}\text { Producto de } \\
\text { intervención/ } \\
\text { control }\end{array}$ & $\begin{array}{l}\text { Cantidad de } \\
\text { beta-glucano } \\
\text { (g/día) }\end{array}$ & $\begin{array}{l}\text { Cambios en } \\
\text { los lípidos } \\
\text { sanguíneos* }\end{array}$ \\
\hline $\begin{array}{l}\text { Van Horn y } \\
\text { Cols. } .^{60}\end{array}$ & 127 & Paralelo & 6 & $\begin{array}{l}\text { CT: } 6,5 \\
\text { LDL: 4,1 } \\
\text { HDL: } 1,7\end{array}$ & $\begin{array}{l}\text { Avena, soja y } \\
\text { avena y leche/ } \\
\text { trigo, soja y } \\
\text { trigo y leche }\end{array}$ & $\begin{array}{l}\text { 6,6-7,9 g/día de } \\
\text { fibra soluble }\end{array}$ & $\begin{array}{l}\downarrow \text { CT: } 3 \% \\
\downarrow \text { LDL: } 5.6 \%\end{array}$ \\
\hline Davy y Cols. ${ }^{61}$ & 36 & Paralelo & 12 & $\begin{array}{c}\text { CT: } 5,1 \\
\text { LDL: } 3,44 \\
\text { HDL: } 0,88 \\
\text { TG: } 1,77\end{array}$ & $\begin{array}{c}\text { Harina de } \\
\text { avena y salvado } \\
\text { avena/grano de } \\
\text { trigo completo }\end{array}$ & 5,5 & $\begin{array}{l}\leftrightarrow \mathrm{CT}^{\mathrm{a}} \\
\downarrow \mathrm{LDL}: 2.5 \% \mathrm{a} \\
\leftrightarrow \mathrm{HDL}^{\mathrm{a}} \\
\leftrightarrow \mathrm{TG}^{\mathrm{a}}\end{array}$ \\
\hline Keenan y Cols. ${ }^{62}$ & 18 & Paralelo & 6 & & $\begin{array}{l}\text { Cereal de } \\
\text { avena/cereal } \\
\text { bajo en fibra }\end{array}$ & 5,52 & $\begin{array}{l}\downarrow \text { CT: } 9 \%^{\mathrm{a}} \\
\downarrow \text { LDL: } 14 \%^{\mathrm{a}}\end{array}$ \\
\hline Pins y Cols. ${ }^{63}$ & 88 & Paralelo & 12 & $\begin{array}{l}\text { CT: } 5,50 \\
\text { LDL: } 3,58\end{array}$ & $\begin{array}{c}\text { Harina de } \\
\text { avena y } \\
\text { cereales } \\
\text { cuadrados de } \\
\text { avena/cereales } \\
\text { de trigo }\end{array}$ & 5,4 & $\begin{array}{l}\downarrow \text { CT: } 11 \% \\
\downarrow \text { LDL: } 12 \%\end{array}$ \\
\hline Kabir y Cols. ${ }^{64}$ & 13 & Cruzado & 4 & CT: 5,4 & $\begin{array}{c}\text { Muesli con } \\
\text { salvado de } \\
\text { avena/dieta } \\
\text { baja en grasa }\end{array}$ & 3 & $\begin{array}{l}\downarrow \mathrm{CT}: 12 \% \\
\uparrow \mathrm{CT}: 4 \% \text { en el } \\
\text { grupo control }\end{array}$ \\
\hline $\begin{array}{l}\text { Kerckhoffs y } \\
\text { Cols. }^{37}\end{array}$ & 25 & Cruzado & 4 & $\begin{array}{l}\text { CT: } 5,58 \\
\text { LDL: 3,77 } \\
\text { HDL: } 1,25\end{array}$ & $\begin{array}{l}\text { Salvado de } \\
\text { avena/fibra de } \\
\text { trigo }\end{array}$ & 5 & $\begin{array}{l}\downarrow C T: 3.9 \% \\
\downarrow L D L: 7 \% \\
\leftrightarrow \text { HDL }\end{array}$ \\
\hline Berg y Cols. ${ }^{65}$ & 235 & Paralelo & 4 & $\begin{array}{c}\text { CT: } 6,69 \\
\text { LDL: } 4,65\end{array}$ & $\begin{array}{l}\text { Dieta + salvado } \\
\text { de avena/dieta } \\
\text { sin salvado de } \\
\text { avena }\end{array}$ & 5 & $\begin{array}{l}\downarrow C T: 4 \% \\
\downarrow \text { LDL: } 6 \% \\
\leftrightarrow \text { HDL, TG }\end{array}$ \\
\hline $\begin{array}{l}\text { Amundsen y } \\
\text { Cols. }{ }^{66}\end{array}$ & 20 & Cruzado & 3 & $\begin{array}{l}\text { CT: } 7,65 \\
\text { LDL: } 5,15 \\
\text { HDL: } 1,71 \\
\text { TG: } 1,96\end{array}$ & $\begin{array}{l}\text { Dieta + salvado } \\
\text { de avena/dieta }\end{array}$ & 5,1 & $\begin{array}{l}\downarrow C T: 6 \% \\
\downarrow \text { LDL: } 9 \% \\
\leftrightarrow \text { HDL, TG }\end{array}$ \\
\hline Frank y Cols. ${ }^{38}$ & 22 & Cruzado & 3 & $\begin{array}{l}\text { CT: 6,2 } \\
\text { LDL: 4,1 }\end{array}$ & $\begin{array}{l}\text { Salvado de } \\
\text { avena de } \\
\text { alto peso } \\
\text { molecular/ } \\
\text { salvado de } \\
\text { avena de bajo } \\
\text { peso molecular }\end{array}$ & 6 & $\begin{array}{l}\downarrow C T: 8.5 \% \\
\downarrow \text { LDL: } 12 \% \\
\text { (sólo en mujeres } \\
\text { en el grupo con } \\
\text { salvado de alto } \\
\text { peso molecular) } \\
\leftrightarrow \text { HDL-c }\end{array}$ \\
\hline $\begin{array}{l}\text { Karmally y } \\
\text { Cols. }{ }^{67}\end{array}$ & 152 & Paralelo & 6 & $\begin{array}{l}\text { CT: } 5,3 \\
\text { LDL: } 3,7\end{array}$ & $\begin{array}{c}\text { Cereales de } \\
\text { desayuno de } \\
\text { avena/cereal } \\
\text { de desayuno de } \\
\text { maíz }\end{array}$ & 3 & $\begin{array}{l}\downarrow C T: \\
5.3 \% \text { a } / 1.3 \% \\
\downarrow L D L: \\
7 \% \text { a } / 2 \%\end{array}$ \\
\hline $\begin{array}{l}\text { Biörklund y } \\
\text { Cols. }^{68}\end{array}$ & 100 & Paralelo & 8 & $\begin{array}{l}\text { CT: } 6,5 \\
\text { LDL: } 4,4 \\
\text { HDL: } 1,4\end{array}$ & $\begin{array}{l}\text { beta-glucano } \\
\text { incorporado en } \\
\text { una bebida/ } \\
\text { bebida con } \\
\text { fibra }\end{array}$ & 5 & $\begin{array}{l}\downarrow C T: 6.4 \% \\
\downarrow L D L: 9 \% \\
\leftrightarrow \text { HDL }\end{array}$ \\
\hline
\end{tabular}


Rev Esp Nutr Hum Diet. 2016; 20(2): 127 - 139

Efectos del consumo del beta-glucano de la avena sobre el colesterol sanguíneo: una revisión

\begin{tabular}{|c|c|c|c|c|c|c|c|}
\hline Referencia & $n$ & $\begin{array}{l}\text { Tipo de } \\
\text { estudio }\end{array}$ & $\begin{array}{l}\text { Duración } \\
\text { del estudio } \\
\text { (semanas) }\end{array}$ & $\begin{array}{l}\text { Lípidos sanguíneos } \\
\text { medios al inicio del } \\
\text { estudio ( } \mathrm{mmol} / \mathrm{L} \text { ) }\end{array}$ & $\begin{array}{c}\text { Producto de } \\
\text { intervención/ } \\
\text { control }\end{array}$ & $\begin{array}{c}\text { Cantidad de } \\
\text { beta-glucano } \\
\text { (g/día) }\end{array}$ & $\begin{array}{l}\text { Cambios en } \\
\text { los lípidos } \\
\text { sanguíneos* }\end{array}$ \\
\hline $\begin{array}{l}\text { Naumann y } \\
\text { Cols. }^{41}\end{array}$ & 47 & Paralelo & 5 & $\mathrm{CT}: \leq 8$ & $\begin{array}{l}\text { beta-glucano } \\
\text { incorporado en } \\
\text { una bebida/ } \\
\text { bebida con } \\
\text { fibra }\end{array}$ & 5 & $\begin{array}{l}\downarrow C T: 4.8 \% \\
\downarrow \text { LDL: } 7.7 \% \\
\leftrightarrow \text { HDL }\end{array}$ \\
\hline Chen y Cols. ${ }^{69}$ & 110 & Paralelo & 12 & $\begin{array}{l}\text { CT: 5,12 } \\
\text { LDL: } 3,20 \\
\text { HDL: } 1,3\end{array}$ & $\begin{array}{c}\text { Magdalenas } \\
\text { y cereales } \\
\text { con salvado } \\
\text { de avena/ } \\
\text { magdalenas } \\
\text { con harina de } \\
\text { trigo refinado } \\
\text { y cereales de } \\
\text { maíz }\end{array}$ & 7 & $\begin{aligned} \leftrightarrow & \mathrm{CT}, \mathrm{LDL}, \\
& \mathrm{HDL}\end{aligned}$ \\
\hline $\begin{array}{l}\text { Theuwissen y } \\
\text { Mensink }{ }^{70}\end{array}$ & 40 & Cruzado & 4 & $\begin{array}{l}\text { CT: } 6,6 \\
\text { LDL: 4,3 }\end{array}$ & $\begin{array}{c}\text { Muesli con } \\
\text { beta-glucano de } \\
\text { avena/muesli } \\
\text { con trigo }\end{array}$ & 5 & $\begin{array}{l}\downarrow C T: 3.4 \% \\
\downarrow L D L: 5 \% \\
\leftrightarrow \text { HDL }\end{array}$ \\
\hline $\begin{array}{l}\text { Queenan y } \\
\text { Cols. }^{42}\end{array}$ & 75 & Paralelo & 6 & $\begin{array}{l}\text { CT: } 6,5 \\
\text { LDL: } 4,4 \\
\text { HDL: } 1,4\end{array}$ & $\begin{array}{l}\text { beta-glucano } \\
\text { incorporado en } \\
\text { una bebida/ } \\
\text { dextrosa }\end{array}$ & 6 & $\begin{array}{l}\downarrow C T: 8.7 \% \\
\downarrow L D L: 9 \% \\
\leftrightarrow \text { HDL }\end{array}$ \\
\hline $\begin{array}{l}\text { Biörklund y } \\
\text { Cols. }^{71}\end{array}$ & 43 & Paralelo & 5 & $\begin{array}{l}\text { CT: } 6,90 \\
\text { LDL: } 4,42\end{array}$ & $\begin{array}{c}\text { Sopa con beta- } \\
\text { glucano/sopa } \\
\text { control }\end{array}$ & 4 & $\leftrightarrow \mathrm{CT}, \mathrm{LDL}$ \\
\hline Liatis y Cols. ${ }^{72}$ & 46 & Paralelo & 3 & $\mathrm{LDL} \geq 3,4$ & $\begin{array}{l}\text { Pan con beta- } \\
\text { glucano/pan sin } \\
\text { beta-glucano }\end{array}$ & 3 & $\begin{array}{l}\downarrow C T: 5 \% \\
\downarrow \text { LDL: } 10 \%\end{array}$ \\
\hline Maki y Cols. $^{73}$ & 204 & Paralelo & 12 & $\begin{array}{l}\text { CT: 5,57 } \\
\text { LDL: 4,01 }\end{array}$ & $\begin{array}{c}\text { Cereales de } \\
\text { desayuno de } \\
\text { grano entero de } \\
\text { avena/cereales } \\
\text { de desayuno } \\
\text { con trigo y maíz } \\
\text { refinados }\end{array}$ & 3 & $\begin{array}{l}\downarrow C T: 2.9 \% \\
\downarrow \text { LDL: } 4.3 \%\end{array}$ \\
\hline Beck y Cols. $^{74}$ & 46 & Paralelo & 12 & $\begin{array}{l}\text { CT: } 5,10 \\
\text { LDL: } 3,04 \\
\text { HDL: } 1,6\end{array}$ & $\begin{array}{l}\text { Salvado de } \\
\text { avena/trigo } \\
\text { refinado y arroz }\end{array}$ & 5,5 & $\begin{aligned} \leftrightarrow & \mathrm{CT}, \mathrm{LDL}, \\
& \mathrm{HDL}\end{aligned}$ \\
\hline $\begin{array}{l}\text { Cugnet-Anceau } \\
\text { y Cols. }{ }^{75}\end{array}$ & 53 & Paralelo & 8 & $\begin{array}{l}\text { CT: } 4,9 \\
\text { LDL: } 2,9 \\
\text { HDL: } 1,2\end{array}$ & $\begin{array}{l}\text { Sopa con beta- } \\
\text { glucano/sopa } \\
\text { control }\end{array}$ & 3,5 & $\begin{aligned} \leftrightarrow & C T, L D L \\
& H D L\end{aligned}$ \\
\hline Wolever y Cols. ${ }^{8}$ & 345 & Paralelo & 4 & $\begin{array}{c}C T: \geq 5 \text { y } \leq 8 \\
\text { LDL: } \geq 3 y \leq 5\end{array}$ & $\begin{array}{c}\text { beta-glucano } \\
\text { de avena a } \\
\text { diferentes } \\
\text { cantidades } \\
\text { y peso } \\
\text { molecular/ } \\
\text { cereales de } \\
\text { desayuno de } \\
\text { trigo }\end{array}$ & $\begin{array}{l}3 \mathrm{~g} \text { de alto PM } \\
4 \mathrm{~g} \text { de } \mathrm{PM} \text { medio } \\
3 \mathrm{~g} \text { de PM medio } \\
4 \mathrm{~g} \text { de bajo PM }\end{array}$ & $\begin{array}{l}\downarrow C T: 4 \% \text { (en } \\
\text { los grupos de } 3 \mathrm{~g} \\
\text { de alto PM y } 4 \mathrm{~g} \\
\text { de PM medio) } \\
\downarrow \text { LDL: } 5,5 \% \text {, } \\
6,5 \% \text { y } 4,7 \% \\
\text { (en los grupos de } \\
3 \mathrm{~g} \text { de alto PM y } \\
\text { de } 3 \text { y } 4 \mathrm{~g} \text { de PM } \\
\text { medio) }\end{array}$ \\
\hline
\end{tabular}




\begin{tabular}{|c|c|c|c|c|c|c|c|}
\hline Referencia & $\mathrm{n}$ & $\begin{array}{l}\text { Tipo de } \\
\text { estudio }\end{array}$ & $\begin{array}{l}\text { Duración } \\
\text { del estudio } \\
\text { (semanas) }\end{array}$ & $\begin{array}{l}\text { Lípidos sanguíneos } \\
\text { medios al inicio del } \\
\text { estudio ( } \mathrm{mmol} / \mathrm{L} \text { ) }\end{array}$ & $\begin{array}{l}\text { Producto de } \\
\text { intervención/ } \\
\text { control }\end{array}$ & $\begin{array}{c}\text { Cantidad de } \\
\text { beta-glucano } \\
\text { (g/día) }\end{array}$ & $\begin{array}{l}\text { Cambios en } \\
\text { los lípidos } \\
\text { sanguíneos* }\end{array}$ \\
\hline $\begin{array}{l}\text { Kristensen y } \\
\text { Bugel }^{76}\end{array}$ & 24 & Cruzado & 2 & CT: 4,03 & $\begin{array}{c}\text { Dieta + salvado } \\
\text { de avena/ dieta } \\
\text { baja en fibra }\end{array}$ & 7,6 & $\downarrow C T: 10 \%$ \\
\hline $\begin{array}{l}\text { Charlton y } \\
\text { Cols. }^{39}\end{array}$ & 62 & Paralelo & 6 & $\begin{array}{l}\text { CT: } 5,90 \\
\text { LDL: } 3,84\end{array}$ & $\begin{array}{c}\text { Salvado de } \\
\text { avena/dieta sin } \\
\text { beta-glucano }\end{array}$ & 3,2 & $\begin{array}{l}\downarrow C T: 7 \% \\
\text { en el grupo de } \\
\text { intervención y } \\
5,5 \% \text { en el grupo } \\
\text { control (respecto } \\
\text { al inicio del } \\
\text { estudio, pero sin } \\
\text { diferencias entre } \\
\text { los grupos) } \\
\downarrow L D L: 8,4 \% \\
\text { en el grupo de } \\
\text { intervención y } \\
5,5 \% \text { en el grupo } \\
\text { control (respecto } \\
\text { al inicio del } \\
\text { estudio, pero sin } \\
\text { diferencias entre } \\
\text { los grupos) }\end{array}$ \\
\hline Zhang y Cols. ${ }^{77}$ & 182 & Paralelo & 6 & $\begin{array}{l}\text { CT: } 6,18 \\
\text { LDL: } 4,24 \\
\text { HDL: } 1,49\end{array}$ & $\begin{array}{c}\text { Cereales } \\
\text { instantáneos de } \\
\text { avena/noodles } \\
\text { de trigo }\end{array}$ & 3,3 & $\begin{array}{l}\downarrow C T: 6,2 \% \\
\text { en el grupo de } \\
\text { intervención } \\
\text { y } 2,3 \% \text { en el } \\
\text { grupo control } \\
\downarrow L D L: 8,4 \% \\
\text { en el grupo de } \\
\text { intervención } \\
\text { y 3,5\% en el } \\
\text { grupo control }\end{array}$ \\
\hline $\begin{array}{l}\text { Ibrügger y } \\
\text { Cols. }^{78}\end{array}$ & 14 & Cruzado & 3 & $\begin{array}{l}\text { CT: } 4,46 \\
\text { LDL: } 2,37\end{array}$ & $\begin{array}{c}\text { Avena/dieta } \\
\text { control }\end{array}$ & 3,3 & $\begin{array}{l}\downarrow C T: 6 \% \\
\downarrow L D L: 12,9 \%\end{array}$ \\
\hline $\begin{array}{l}\text { Thongoun y } \\
\text { Cols. }^{79}\end{array}$ & 24 & Cruzado & 8 & & $\begin{array}{c}\text { Cereales de } \\
\text { avena/cereales } \\
\text { de arroz }\end{array}$ & 3 & $\begin{array}{l}\downarrow C T: 5 \% \\
\downarrow L D L: 10 \%\end{array}$ \\
\hline
\end{tabular}

CT: colesterol total; LDL: Lipoproteínas de baja densidad; HDL: Lipoproteínas de alta densidad; TG: Triglicéridos; $\downarrow$ : disminución; $\leftrightarrow$ : sin variación.

* Diferencias respecto al grupo al grupo control; a: diferencias encontradas con respecto al inicio del estudio en el grupo de intervención.

Un 78\% de los estudios encontraron efectos hipocolesterolemiantes del beta-glucano de avena, lo que coincide con la revisión realizada por Othman y Cols. ${ }^{32}$. La reducción del CT observado varió del 2,9 al 12\% y la reducción de LDL del 2,0 al 12,9\% (Tabla 2), no habiéndose encontrado cambios significativos en la concentración sérica de las lipoproteínas de alta densidad (HDL) o de los triglicéridos como marcadores asociados al riesgo de enfermedad cardiovascular. Thies y Cols. ${ }^{80}$ en una revisión sistemática publicada en 2014 indicaron que el consumo regular de avena, o del salvado de avena, tiene un efecto beneficioso sobre el colesterol sanguíneo, observando disminuciones del 3-6\% para el CT y del 4-8\% para las $L D L$, por lo que se concluye que el consumo de beta-glucano de avena tiene efectos reductores sobre las cifras sanguíneas de colesterol, especialmente en individuos hipercolesterolémicos. Es generalmente aceptado que la reducción de un $1 \%$ de las cifras del CT o de las LDL se asocia con una disminución del riesgo de enfermedad coronaria del 2-3\% y $1 \%$, respectivamente ${ }^{81}$. 
Estos resultados coinciden con diversos meta-análisis y revisiones sistemáticas realizados hasta el momento. Ripsin y Cols. ${ }^{82}$ concluyeron que el consumo de $3 \mathrm{~g} /$ día de beta-glucano de avena disminuye las cifras de colesterol en 0,13 a 0,16mmol/L. Tiwari y Col.7 señalaron que el consumo de $3 \mathrm{~g} /$ día de betaglucano de avena, o de cebada, es suficiente para disminuir el colesterol en sangre, observando disminuciones de $0,60 \mathrm{mmol} / \mathrm{L}$ en el CT y de $0,66 \mathrm{mmol} / \mathrm{L}$ para las LDL y, en el caso concreto de la avena reducciones de $0,53 \mathrm{mmol} / \mathrm{L}$ y $0,56 \mathrm{mmol} / \mathrm{L}$, respectivamente. Whitehead y Cols. ${ }^{6}$ indicaron que la inclusión de $\geq 3 \mathrm{~g} /$ día de beta-glucano de avena a la dieta se asoció a reducciones del CT en $0,30 \mathrm{mmol} / \mathrm{L}$ y de las LDL en $0,25 \mathrm{mmol} / \mathrm{L}$. Zhu y Cols. ${ }^{83}$ concluyeron que el consumo de beta-glucano de avena, o de cebada, disminuye el CT en $0,26 \mathrm{mmol} / \mathrm{L}$ y las $L D L$ en $0,21 \mathrm{mmol} / \mathrm{L}$, observando en el caso de la avena reducciones de $0,24 \mathrm{mmol} / \mathrm{L}$ y $0,20 \mathrm{mmol} / \mathrm{L}$, respectivamente. En ninguno de los metaanálisis mencionados se encontró que el consumo de avena o beta-glucano de avena modifique las concentraciones séricas de las HDL o de los triglicéridos. Aunque las reducciones en las cifras de colesterol sanguíneo puedan considerarse como moderadas, un aumento de $0,26 \mathrm{mmol} / \mathrm{L}$ de las $L D L$ se asocia con un incremento del riesgo cardiovascular del $12 \%{ }^{84}$.

\section{CONCLUSIONES}

Debido a la elevada prevalencia de la enfermedad coronaria y al elevado coste que supone la medicación para el tratamiento de la misma, junto a una modificación del estilo de vida (que debe incluir modificación de actividad física y dieta), el consumo de alimentos funcionales puede contribuir a controlarla. Numerosos estudios avalan que la inclusión en la dieta, de forma regular, de al menos $3 \mathrm{~g}$ de beta-glucano de avena tiene efectos beneficiosos sobre las cifras de colesterol en sangre en la población, especialmente en las personas con hipercolesterolemia. El efecto reductor del colesterol sanguíneo del beta-glucano se debe, principalmente, a su capacidad para disminuir la absorción de colesterol dietético y la recaptación de los ácidos biliares. Debido a que las características físico-químicas del beta-glucano, así como su peso molecular, determinan las propiedades fisiológicas del mismo en el organismo, sería deseable que en futuras investigaciones se tengan en cuenta estos factores.

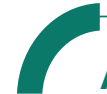

\section{AGRADECIMIENTOS}

R. M. O. y A. A. han contribuido por igual en la recopilación de información, en la interpretación de los resultados y en la redacción del artículo.

\section{CONFLICTO DE INTERESES \\ CONFLICTO DE INTERESES}

Artículo realizado con el apoyo económico de Adam Foods S.L.U. Adam Foods S.L.U. no ha tenido participación en la metodología de revisión, la recopilación de datos, el análisis o la interpretación de estos, ni en la redacción del manuscrito.

\section{FINANCIACIÓN}

Adam Foods S.L.U.

\section{REFERENCIAS}

(1) Clemens R, van Klinken BJ. Oats, more than just a whole grain: an introduction. Br] Nutr. 2014; 112(Suppl 2): S1-3. doi: 10.1017/S0007114514002712

(2) Singh R, De S, Belkheir A. Avena sativa (Oat), a potential neutraceutical and therapeutic agent: an overview. Crit Rev Food Sci Nutr. 2013; 53(2): 126-44. doi: 10.1080/10408398.2010.526725

(3) Koskinen O, Villanen M, Korponay-Szabo I, Lindfors K, Maki $M$, Kaukinen K. Oats do not induce systemic or mucosal autoantibody response in children with coelical disease. ] Pediatr Gastroenterol Nutr. 2009; 48(5): 559-65. doi: 10.1097/ MPG.0b013e3181668635

(4) Sadiq Butt M, Tahir-Nadeem M, Khan MK, Shabir R, Butt MS. Oat: unique among the cereals. Eur ] Nutr. 2008; 47(2): 68-79. doi: 10.1007/s00394-008-0698-7

(5) Ortega RM, Aparicio Vizuete A, Jimenez Ortega Al, Rodriguez Rodriguez E. [Wholegrain cereals and sanitary benefits]. Nutr Hosp. 2015; 32(Suppl 1): 25-31. doi: 10.3305/nh.2015.32. sup1.9475

(6) Whitehead A, Beck EJ, Tosh S, Wolever TM. Cholesterol-lowering effects of oat beta-glucan: a meta-analysis of randomized controlled trials. Am J Clin Nutr. 2014; 100(6): 1413-21. doi: 10.3945/ajcn.114.086108 
(7) Tiwari U, Cummins E. Meta-analysis of the effect of beta-glucan intake on blood cholesterol and glucose levels. Nutrition. 2011; 27(10): 1008-16. doi: 10.1016/j.nut.2010.11.006

(8) Wolever TM, Tosh SM, Gibbs AL, Brand-Miller J, Duncan A M, Hart V, et al. Physicochemical properties of oat beta-glucan influence its ability to reduce serum LDL cholesterol in humans: a randomized clinical trial. Am J Clin Nutr. 2010; 92(4): 723-32. doi: 10.3945/ajcn.2010.29174

(9) United States Department of Agriculture (USDA). Dietary Guidelines for Americans,2010 [portal en internet]. Estados Unidos; USDA; 2011; [citado 17 Ago 2015]. Disponible en: http://www.cnpp.usda.gov/publications/ dietaryguidelines/2010/policydoc/policydoc.pdf

(10) Aparicio A, Ortega RM, Requejo AM. Guías en alimentación: consumo aconsejado de alimentos. En: Ortega RM, Requejo AM, editores. Nutriguía. Manual de Nutrición Clínica. Madrid: Editorial Médica Panamericana; 2015. p. 27-42.

(11) Volman JJ, Mensink RP, Ramakers JD, de Winther MP, Carlsen $H$, Blomhoff $R$, et al. Dietary $(1 \rightarrow 3),(1 \rightarrow 4)$-beta-D-glucans from oat activate nuclear factor-kappaB in intestinal leukocytes and enterocytes from mice. Nutr Res. 2010; 30(1): 40-8. doi: 10.1016/j.nutres.2009.10.023

(12) Cui W, Wood PJ, Blackwell B, Nikiforuk J. Physicochemical properties and structural characterization by two-dimensional NMR spectroscopy of wheat $\beta$-D-glucan-comparison with other cereal $\beta$-D-glucans. Carbohydr Polym. 2000; 41(3): 24958. doi: 10.1016/S0144-8617(99)00143-5

(13) Miller SS, Fulcher RG, Sen A, Arnason JT. Oat endosperm cell walls. Isolation, compostition and comparison with other tissues. Cereal Chem. 1995; 72: 421-7.

(14) Wang Q, Ellis PR. Oat beta-glucan: physico-chemical characteristics in relation to its blood-glucose and cholesterollowering properties. Br ] Nutr. 2014; 112(Suppl 2): S4-13. doi: 10.1017/S0007114514002256

(15) Miller SS, Wood PJ, Pietrzak LN, Flucher RG. Mixed linkage $\beta$-glucan, protein content, and kernel weight in Avena species. Cereal Chem. 1993; 70(2): 231-3.

(16) Ajithkumar A, Andersson R, Aman P. Content and molecular weight of extractable beta-glucan in American and Swedish oat samples. J Agric Food Chem. 2005; 53(4): 1205-9. doi: 10.1021/jf040322c

(17) Peterson DM, Wesenberg DM, Burrup DE. $\beta$-Glucan Content and Its Relationship to Agronomic Characteristics in Elite Oat Germplasm. Crop Sci. 1995; 35(4): 965-70. doi: 10.2135/crops ci1995.0011183X003500040005x

(18) Stuart IM, Loi L, Fincher GB. Inmunological comparison of $(1 \rightarrow 3$, $1 \rightarrow 4)$-b-glucan endohydrolases in germinating cereals. J Cereal Sci. 1987; 6(1): 45-52. doi: 10.1016/0014-5793(82)80602-9

(19) Áman P, Rimsten L, Andersson R. Molecular weight distribution of beta-glucan in oat-based foods. Cereal Chem. 2004; 81(3): 356-60. doi: 10.1094/CCHEM.2004.81.3.356
(20) Decker EA, Rose DJ, Stewart D. Processing of oats and the impact of processing operations on nutrition and health benefits. Br ] Nutr. 2014; 112(Suppl 2): S58-64. doi: 10.1017/ S000711451400227X

(21) Saastamiunen M, Hietaniemi V, Pihlava JM. $\beta$-Glucan contents of groats of different oat cultivars in official variety, in organic cultivation, and in nitrogen fertilization trials in Finland. Agr Food Sci. 2004; 13(1-2): 68-79. doi: 10.2137/1239099041838076

(22) Dvončová D, Havrlentová M, Hlinková A, Hozlár P. Effect of fertilization and variety on the $\beta$-glucan content in the grain of oats. Food Sci Tech Quality. 2010; 17(70): 108-16

(23) Wood PJ. Cereal $\beta$-glucans in diet and health. J Cereal Sci. 2007; 46(3): 230-8.

(24) Erkkila AT, Lichtenstein AH. Fiber and cardiovascular disease risk: how strong is the evidence? J Cardiovasc Nurs. 2006; 21(1): 3-8.

(25) Andersson M, Ellegard L, Andersson H. Oat bran stimulates bile acid synthesis within $8 \mathrm{~h}$ as measured by 7alpha-hydroxy4-cholesten-3-one. Am J Clin Nutr. 2002; 76(5): 1111-6.

(26) Kim HJ, White PJ. Interactional effects of beta-glucan, starch, and protein in heated oat slurries on viscosity and in vitro bile acid binding. J Agric Food Chem. 2012; 60(24): 6217-22. doi: 10.1021/jf300786f

(27) European Food Safety Agency (EFSA). Scientific Opinion on the substantiation of a health claim related to oat beta glucan and lowering blood cholesterol and reduced risk of (coronary) heart disease pursuant to Article 14 of Regulation (EC) No 1924/2006. EFSA J. 2010: 1885-99.

(28) Food and Drug Administration (FDA). Soluble fibre from whole oats and coronary heart disease health claim Fed Regist. 1997; 62: 3583-601.

(29) Wang Q, Ellis PR, Ross-Murphy SB. Dissolution kinetics of guargum powders-II. Efects of concentration and molecular weight. Carbohydr Polym. 2003; 53(1): 75-83. doi:10.1016/S01448617(03)00009-2

(30) Lazaridou A, Biliaderis CG, Izydorrczyk MS. Molecular size effects on rheological properties of beta-glucans in solution and gels. Food Hydrocolloid. 2003; 17: 693-712.

(31) Kim SY, Song HJ, Lee YY, Cho KH, Roh YK. Biomedical issues of dietary fiber beta-glucan. J Korean Med Sci. 2006; 21(5): 781-9. doi: 10.3346/jkms.2006.21.5.781

(32) Othman RA, Moghadasian MH, Jones PJ. Cholesterol-lowering effects of oat $\beta$-glucan. Nutr Rev. 2011; 69(6): 299-309. doi: 10.1111/j.1753-4887.2011.00401.x

(33) Serna-Saldivar SO. Cereal Grains: Properties, Processing, and Nutritional Attributes. Monterrey, Mexico: Bota Raton, FL; 2010.

(34) Tosh SM, Brummer Y, Miller SS, Regand A, Defelice C, Duss $R$, et al. Processing affects the physicochemical properties of beta-glucan in oat bran cereal. J Agric Food Chem. 2010; 58(13): 7723-30. doi: 10.1021/jf904553u 
(35) Zhang M, Bai X, Zhang Z. Extrusion process improves the functionality of soluble dietary fiber in oat bran. J Cereal Sci. 2011; 54(1): 98-103. doi:10.1016/j.jcs.2011.04.001

(36) Regand A, Tosh SM, Wolever TM, Wood PJ. Physicochemical properties of beta-glucan in differently processed oat foods influence glycemic response. J Agric Food Chem. 2009; 57(19): 8831-8. doi: 10.1021/jf901271v

(37) Kerckhoffs DA, Hornstra G, Mensink RP. Cholesterollowering effect of beta-glucan from oat bran in mildly hypercholesterolemic subjects may decrease when betaglucan is incorporated into bread and cookies. Am ] Clin Nutr. 2003; 78(2): 221-7.

(38) Frank J, Sundberg B, Kamal-Eldin A, Vessby B, Aman P. Yeastleavened oat breads with high or low molecular weight betaglucan do not differ in their effects on blood concentrations of lipids, insulin, or glucose in humans. J Nutr. 2004; 134(6): 1384-8.

(39) Charlton KE, Tapsell LC, Batterham MJ, O'Shea J, Thorne $R$, Beck $E$, et al. Effect of 6 weeks' consumption of betaglucan-rich oat products on cholesterol levels in mildly hypercholesterolaemic overweight adults. Br J Nutr. 2012; 107(7): 1037-47. doi: 10.1017/S0007114511003850

(40) Onning G, Wallmark A, Persson M, Akesson B, Elmstahl S, Oste R. Consumption of oat milk for 5 weeks lowers serum cholesterol and LDL cholesterol in free-living men with moderate hypercholesterolemia. Ann Nutr Metab. 1999; 43(5): 301-9.

(41) Naumann E, van Rees AB, Onning G, Oste R, Wydra M, Mensink RP. Beta-glucan incorporated into a fruit drink effectively lowers serum LDL-cholesterol concentrations. Am J Clin Nutr. 2006; 83(3): 601-5.

(42) Queenan KM, Stewart ML, Smith KN, Thomas W, Fulcher RG, Slavin JL. Concentrated oat beta-glucan, a fermentable fiber, lowers serum cholesterol in hypercholesterolemic adults in a randomized controlled trial. Nutr J. 2007; 6: 6. doi: 10.1186/1475-2891-6-6

(43) Beer MU, Wood PJ, Weisz J, Fillion N. Effect of cooking and storage on the amount and molecular weight of $(1 \rightarrow 3)$ $(1 \rightarrow 4)-\beta$-D-glucan extracted from oat products by an in vitro digestion system. Cereal Chem. 1997; 74(6): 705-9. doi: 10.1094/CCHEM.1997.74.6.705

(44) Lan-Pidhainy X, Brummer Y, Tosh SM, Wolever TM, Wood PJ. Reducing beta-glucan solubility in oat bran muffins by freezethaw treatment attenuates its hypoglycemic effect. Cereal Chem. 2007;84(5):512-7. doi: 10.1094/CCHEM-84-5-0512

(45) Chen J, Huang XF. The effects of diets enriched in beta-glucans on blood lipoprotein concentrations. J Clin Lipidol. 2009; 3(3): 154-8. doi: 10.1016/j.jacl.2009.04.054

(46) Ellegard L, Andersson H. Oat bran rapidly increases bile acid excretion and bile acid synthesis: an ileostomy study. Eur ] Clin Nutr. 2007; 61(8): 938-45. doi: 10.1038/sj.ejcn.1602607
(47) Reihner E, Angelin B, Rudling M, Ewerth S, Bjorkhem I, Einarsson K. Regulation of hepatic cholesterol metabolism in humans: stimulatory effects of cholestyramine on HMGCoA reductase activity and low density lipoprotein receptor expression in gallstone patients. J Lipid Res. 1990; 31(12): 2219-26.

(48) Murray CJ, Lopez AD. Mortality by cause for eight regions of the world: Global Burden of Disease Study. Lancet. 1997; 349(9061): 1269-76. doi: 10.1016/S0140-6736(96)07493-4

(49) Reiner Z, Catapano AL, De Baker G, Graham I, Taskinen MR, Wiklund 0 , et al. ESC/EAS Guidelines for the management of dyslipidaemias: the Task Force for the management of dyslipidaemias of the European Society of Cardiology (ESC) and the European Atherosclerosis Society (EAS). Eur Heart ]. 2011; 32(14): 1769-818. doi: 10.1093/eurheartj/ehr158

(50) Expert Panel on Detection E, Treatment of High Blood Cholesterol in A. Executive Summary of The Third Report of The National Cholesterol Education Program (NCEP) Expert Panel on Detection, Evaluation, And Treatment of High Blood Cholesterol In Adults (Adult Treatment Panel III). JAMA. 2001; 285(19): 2486-97. doi: 10.1001/jama.285.19.2486

(51) Ballantyne C, Arroll B, Shepherd J. Lipids and CVD management: towards a global consensus. Eur Heart J. 2005; 26(21): 222431. doi: 10.1093/eurheartj/ehi373

(52) Bazzano LA, He J, Ogden LG, Loria CM, Whelton PK; National Health and Nutrition Examination Survey I Epidemiologic Follow-up Study. Dietary fiber intake and reduced risk of coronary heart disease in US men and women: the National Health and Nutrition Examination Survey I Epidemiologic Follow-up Study. Arch Intern Med. 2003; 163(16): 1897-904. doi: 10.1001/archinte.163.16.1897

(53) Threapleton DE, Greenwood DC, Evans CE, Cleghorn C L, Nykjaer C, Woodhead C, et al. Dietary fibre intake and risk of cardiovascular disease: systematic review and meta-analysis. BM]. 2013; 347: f6879. doi: 10.1136/bmj.f6879

(54) Chen ZY, Jiao R, Ma KY. Cholesterol-lowering nutraceuticals and functional foods. J Agric Food Chem. 2008; 56(19): 876173. doi: 10.1021/jf801566r

(55) Bureau of Nutritional Sciences FD, Health Products and Food Branch, Health Canada. Oat products and blood cholesterol lowering: summary of assessment of a health claim about oat products and blood cholesterol lowering [portal en internet]. Ottawa, Ontario: Health Canada; 2010 [actualizado 25 Nov 2010; citado 23 Agos 2015]. Disponible en: http://www.hc-sc. gc.ca/fn-an/alt_formats/pdf/label-etiquet/claims-reclam/ assess-evalu/oat_avoine-eng.pdf

(56) Food Standards Australian and New Zealand. Standard 1.2.7: Nutrition, health and related claims. Food Standards Gazette [revista en internet]. 2013 [consulta 25/08/2015]. Disponible en: http://www. foodstandards.gov.au/code/changes/ gazette_138.pdf 
(57) Ministry of Health Malaysia. Malaysian dietary guidelines-Key Message14-make effective use of nutrition information on food labels [portal en internet]. Putrajaya, Malasia: Ministry of Health Malaysia; 2010 [actualizado 27 Nov 2011; citado 24 Agos 2015]. Disponible en: http://www2.moh.gov.my/images/ gallery/Garispanduan/diet/km14.pdf

(58) Joint Health Claims Initiative. Final report on a Generic health claim for oats and reduction of blood cholesterol [portal en internet]. Reino Unido; 2004 [actualizado 2 Jun 2006, citado 24 Agos 2015]. Disponible en: http://webarchive. nationalarchives.gov.uk/nobanner/20130404135254/http:/ www.jhci.org.uk/approv/oats.htm

(59) Lovegrove JA, Clohessy A, Milon H, Williams CM. Modest doses of beta-glucan do not reduce concentrations of potentially atherogenic lipoproteins. Am J Clin Nutr. 2000; 72(1): 49-55.

(60) Van Horn L, Liu K, Gerber J, Garside D, Schiffer L, Gernhofer $\mathrm{N}$, et al. Oats and soy in lipid-lowering diets for women with hypercholesterolemia: is there synergy? ] Am Diet Assoc. 2001; 101(11): 1319-25. doi: 10.1016/S0002-8223(01)00317-0

(61) Davy BM, Davy KP, Ho RC, Beske SD, Davrath LR, Melby CL. High-fiber oat cereal compared with wheat cereal consumption favorably alters $\mathrm{LDL}$-cholesterol subclass and particle numbers in middle-aged and older men. Am J Clin Nutr. 2002; 76(2): 351-8.

(62) Keenan JM, Pins J], Frazel C, Moran A, Turnquist L. Oat ingestion reduces systolic and diastolic blood pressure in patients with mild or borderline hypertension: a pilot trial. ] Fam Pract. 2002; 51(4): 369

(63) Pins J], Geleva D, Keenan JM, Frazel C, O'Connor PJ, Cherney LM. Do whole-grain oat cereals reduce the need for antihypertensive medications and improve blood pressure control? J Fam Pract. 2002; 51(4): 353-9.

(64) Kabir M, Oppert JM, Vidal H, Bruzzo F, Fiquet C, Wursch P, et al. Four-week low-glycemic index breakfast with a modest amount of soluble fibers in type 2 diabetic men. Metabolism. 2002; 51(7): 819-26. doi: 10.1053/meta.2002.33345

(65) Berg A, Konig D, Deibert P, GrathwohI D, Berg A, Baumstark M $W$, et al. Effect of an oat bran enriched diet on the atherogenic lipid profile in patients with an increased coronary heart disease risk. A controlled randomized lifestyle intervention study. Ann Nutr Metab. 2003; 47(6): 306-11. doi: 10.1159/000072404

(66) Amundsen AL, Haugum B, Andersson H. Changes in serum cholesterol and sterol metabolites after intake of products enriched with an oat bran concentrate within a controlled diet. Scand J Nutr. 2003; 47(2): 68-74. doi: 10.1080/11026480310009573

(67) Karmally W, Montez MG, Palmas W, Martinez W, Branstetter A, Ramakrishnan $R$, et al. Cholesterol-lowering benefits of oat-containing cereal in Hispanic americans. J Am Diet Assoc. 2005; 105(6): 967-70. doi: 10.1016/j.jada.2005.03.006
(68) Biörklund M, van Rees A, Mensink RP, Onning G. Changes in serum lipids and postprandial glucose and insulin concentrations after consumption of beverages with betaglucans from oats or barley: a randomised dose-controlled trial. Eur ] Clin Nutr. 2005; 59(11): 1272-81. doi:10.1038/ sj.ejcn. 1602240

(69) Chen J, He J, Wildman RP, Reynolds K, Streiffer RH, Whelton PK. A randomized controlled trial of dietary fiber intake on serum lipids. Eur ] Clin Nutr. 2006; 60(1): 62-8. doi:10.1038/ sj.ejcn.1602268

(70) Theuwissen E, Mensink RP. Simultaneous intake of beta-glucan and plant stanol esters affects lipid metabolism in slightly hypercholesterolemic subjects. J Nutr. 2007; 137(3): 583-8.

(71) Biörklund M, Holm J, Onning G. Serum lipids and postprandial glucose and insulin levels in hyperlipidemic subjects after consumption of an oat beta-glucan-containing ready meal. Ann Nutr Metab. 2008; 52(2): 83-90. doi: 10.1159/000121281

(72) Liatis S, Tsapogas P, Chala E, Dimosthenopoulos C, Kyriakopoulos K, Kapantais $E$, et al. The consumption of bread enriched with betaglucan reduces LDL-cholesterol and improves insulin resistance in patients with type 2 diabetes. Diabetes Metab. 2009; 35(2): 115-20. doi: 10.1016/j. diabet.2008.09.004

(73) Maki KC, Beiseigel JM, Jonnalagadda SS, Gugger CK, Reeves MS, Farmer MV, et al. Whole-grain ready-to-eat oat cereal, as part of a dietary program for weight loss, reduces low-density lipoprotein cholesterol in adults with overweight and obesity more than a dietary program including low-fiber control foods. J Am Diet Assoc. 2010; 110(2): 205-14. doi: 10.1016/j. jada.2009.10.037

(74) Beck EJ, Tapsell LC, Batterham MJ, Tosh SM, Huang XF. Oat betaglucan supplementation does not enhance the effectiveness of an energy-restricted diet in overweight women. $\mathrm{Br}]$ Nutr. 2010; 103(8): 1212-22. doi: 10.1017/S0007114509992856

(75) Cugnet-Anceau C, Nazare JA, Biörklund M, Le Coquil E, Sassolas A, Sothier $M$, et al. A controlled study of consumption of betaglucan-enriched soups for 2 months by type 2 diabetic freeliving subjects. Br J Nutr. 2010; 103(3): 422-8. doi: 10.1017/ S0007114509991875

(76) Kristensen M, Bugel S. A diet rich in oat bran improves blood lipids and hemostatic factors, and reduces apparent energy digestibility in young healthy volunteers. Eur ] Clin Nutr. 2011; 65(9): 1053-8. doi: 10.1038/ejcn.2011.102

(77) Zhang J, Li L, Song P, Wang C, Man Q, Meng L, et al. Randomized controlled trial of oatmeal consumption versus noodle consumption on blood lipids of urban Chinese adults with hypercholesterolemia. Nutr J. 2012; 11: 54. doi: 10.1186/14752891-11-54

(78) Ibrügger S, Kristensen M, Poulsen MW, Mikkelsen MS, Ejsing J, Jespersen BM, et al. Extracted oat and barley beta-glucans do 
not affect cholesterol metabolism in young healthy adults. ] Nutr. 2013; 143(10): 1579-85. doi: 10.3945/jn.112.173054

(79) Thongoun P, Pavadhgul P, Bumrungpert A, Satitvipawee P, Harjani Y, Kurilich A. Effect of oat consumption on lipid profiles in hypercholesterolemic adults. ] Med Assoc Thai. 2013; 96(Suppl 5): S25-32.

(80) Thies F, Masson LF, Boffetta P, Kris-Etherton P. Oats and CVD risk markers: a systematic literature review. $\mathrm{Br}$ ] Nutr. 2014; 112(Suppl 2): S19-30. doi: 10.1017/S0007114514002281

(81) National Cholesterol Education Program Expert Panel on Detection E, Treatment of High Blood Cholesterol in A. Third Report of the National Cholesterol Education Program (NCEP) Expert Panel on Detection, Evaluation, and Treatment of High Blood Cholesterol in Adults (Adult Treatment Panel III) final report. Circulation. 2002; 106(25): 3143-421.
(82) Ripsin CM, Keenan JM, Jacobs DR, Jr., Elmer P J, Welch $R R$, Van Horn $L$, et al. Oat products and lipid lowering. $A$ meta-analysis. JAMA. 1992; 267(24): 3317-25. doi:10.1001/ jama.1992.03480240079039

(83) Zhu X, Sun X, Wang M, Zhang C, Cao Y, Mo G, et al. Quantitative assessment of the effects of beta-glucan consumption on serum lipid profile and glucose level in hypercholesterolemic subjects. Nutr Metab Cardiovasc Dis. 2015; 25(8): 714-23. doi: 10.1016/j.numecd.2015.04.008

(84) Horward BV, Robbins DC, Sievers ML, Lee ET, Rhoades D, Devereux RB, et al. LDL cholesterol as a strong predictor of coronary heart disease in diabetic individuals with insulin resistance and low LDL: the Strong Heart Study. Arterioscler Thromb Vasc Biol. 2000; 20(3): 830-5. 